\title{
Produção, decomposição e qualidade nutricional da serapilheira foliar em uma floresta paludosa de altitude
}

\author{
Vinicius Leandro Terror ${ }^{1,2}$, Hildeberto Caldas de Sousa ${ }^{1}$ e Alessandra Rodrigues Kozovits ${ }^{1}$
}

Recebido em 10/03/2010. Aceito em 16/12/2010

\section{RESUMO}

(Produção, decomposição e qualidade nutricional da serapilheira foliar em uma floresta paludosa de altitude). Florestas paludosas são ecossistemas com ocorrência restrita a solos hidromórficos, sujeitos à presença superficial de água devido ao afloramento do lençol freático, onde a diversidade e o estabelecimento da vegetação dependem de sua adaptabilidade às condições de saturação hídrica e baixa oxigenação do solo. Este estudo teve como objetivo caracterizar aspectos da ciclagem de N e P em uma floresta paludosa no Parque Estadual do Itacolomi, MG, através de mensurações da produção, qualidade nutricional e decomposição da serapilheira foliar durante o período de outubro de 2006 a setembro de 2007. A produção anual média de serapilheira não apresentou variações espaciais e foi de $5.7 \mathrm{t} \mathrm{ha}^{-1}$, com pico de produção em setembro. A fração foliar foi responsável por $57 \%$ da produção total de serapilheira e apresentou concentrações de N, P e K de 12,6; 0,616 e 3,07 $\mathrm{g} \mathrm{kg}^{-1}$, respectivamente. A porcentagem de perda de massa durante a decomposição de serapilheira foi inversamente correlacionada com o conteúdo de água no solo, variando de $29,2 \%$ a $46,2 \%$. A razão N:P da serapilheira foliar sugere que a vegetação cresça sob limitação por P. Considerando-se os parâmetros avaliados, a floresta paludosa apresentou um taxa de ciclagem de nutrientes lenta, principalmente em razão da baixa qualidade nutricional da serapilheira e sua lenta decomposição, em comparação com valores obtidos em outros estudos em ecossistemas florestais tropicais úmidos.

Palavras-chave: ciclagem de nutrientes, florestas inundáveis, razão N:P, solos hidromórficos

\begin{abstract}
(Nutrient cycling in a high-altitude swamp forest). Swamp forests are ecosystems restricted to hydromorphic soils which are subject to the presence of surface water due to upwelling of groundwater. The diversity and establishment of plant communities depend on adaptability to conditions of flooding and low oxygen in the soil. This study aimed to characterize aspects of $\mathrm{N}$ and $\mathrm{P}$ cycling in a swamp forest in Itacolomi Park, Minas Gerais, Brazil, by measuring parameters such as litterfall, nutrient quality and decomposition of leaf litter during the period October 2006 to September 2007. Mean annual litter production was $5.7 \mathrm{t} \mathrm{ha}^{-1}$, with peak production in September. The leaf fraction accounted for $57 \%$ of total litterfall, and concentrations of $\mathrm{N}, \mathrm{P}$ and $\mathrm{K}$ were $12.6,0.616$ and $3.07 \mathrm{~g} \mathrm{~kg}^{-1}$, respectively. Leaf-mass loss during litter decomposition was negatively correlated to soil water content, varying between 29.2 and $46.2 \%$. The N:P ratio of leaf litter suggests that the vegetation grows under P-limitation. Taking into account the evaluated parameters, the swamp forest showed a slower rate of nutrient cycling, mainly due to low litter nutrient quality and slower litter decomposition rate when compared with other wet tropical forests.
\end{abstract}

Key words: hydromorphic soils, nutrient cycling, N:P ratio, swamp forests

\section{Introdução}

As florestas paludosas são formações com ocorrência restrita a solos hidromórficos, sujeitos à presença superficial de água em caráter temporário ou permanente devido ao afloramento do lençol freático (Torres et al. 1994; Toniato et al. 1998), o que confere ao ambiente o aspecto geral conhecido como "brejo". Em geral, o estabelecimento da vegetação nas florestas paludosas e áreas úmidas é fortemente regu- lado pela freqüência, intensidade e duração da inundação, conjunto de fatores que confere a esses ecossistemas um alto grau de saturação hídrica e baixa oxigenação do solo (Harms et al. 1980).

Durante as últimas décadas, as áreas úmidas, dentre elas as florestas paludosas, vêm sofrendo um intenso processo de degradação, especialmente pela drenagem de seus solos e transformação em terras agrícolas, industriais e urbanas, e estão desaparecendo sem que se conheçam suas

1 Universidade Federal de Ouro Preto, Departamento de Biodiversidade, Evolução e Meio Ambiente, Campus Morro do Cruzeiro, Ouro Preto, MG, Brasil

2 Autor para correspondência: viniciusterror@yahoo.com 
funções ecológicas e sua importância para a manutenção dos recursos hídricos (Richardson \& Vepraskas 2001). A composição florística e a estrutura fitossociológica das florestas paludosas do sudeste do Brasil têm sido amplamente estudadas (Torres et al. 1994; Teixeira \& Assis. 2005), mas pouco se conhece acerca dos principais processos ecológicos inerentes à interação solo-planta nesses ecossistemas, como a ciclagem de nutrientes. Os processos relacionados à ciclagem de nutrientes têm sido estudados em uma vasta gama de ambientes, como florestas tropicais úmidas da América do Sul (Borém \& Ramos 2002; Pinto et al. 2009), da América Central (Cuevas \& Lugo 1998), da Ásia (Dent et al. 2006) e da África (Greenland \& Kowal 1960), além de matas ciliares e florestas ripárias brasileiras (Pagano \& Durigan 2000; Vital et al. 2004) e florestas úmidas na Amazônia (Villela \& Proctor 1999; Barlow et al. 2007). Entretanto, não foram encontrados registros de estudos dessa natureza em florestas paludosas brasileiras.

A produtividade em ecossistemas terrestres está diretamente ligada à ciclagem de nutrientes entre os diversos componentes do sistema solo-planta. Em outras palavras, quando nutrientes são continuamente extraídos do solo pelas plantas e microrganismos ou transferidos para outros compartimentos do ecossistema, a velocidade com que essas perdas ocorrem é um fator chave para o ajuste da produtividade vegetal (Vitousek 1982). Em grande parte dos ecossistemas florestais, a ciclagem de nutrientes ocorre principalmente através da deposição de serapilheira, que determina a quantidade de nutrientes liberados pela vegetação, enquanto sua decomposição caracteriza o retorno efetivo desses nutrientes ao solo (Vitousek 1984; Montagnini \& Jordan 2005). Devido à sua importância para o equilíbrio dos ecossistemas florestais, tais processos têm sido amplamente adotados como indicadores da integridade ecológica desses ecossistemas, uma vez que além do seu papel na ciclagem de nutrientes, a serapilheira confere heterogeneidade espacial e temporal ao ambiente, além de servir como recurso para uma grande diversidade de microrganismos e invertebrados (Vitousek \& Sanford Jr 1986; Arato et al. 2003).

Este estudo teve como objetivo geral caracterizar a produção, qualidade nutricional e decomposição da serapilheira foliar em uma floresta paludosa de altitude com a intenção de ampliar os conhecimentos sobre os principais processos ecológicos ligados à ciclagem de nutrientes desse tipo de ecossistema, fornecendo dados para a conservação e manejo das áreas úmidas florestais.

\section{Materiais e métodos}

\section{Área de estudo}

O Parque Estadual do Itacolomi (PEIT) é uma Unidade de Conservação de proteção integral sob responsabilidade do Instituto Estadual de Florestas (IEF/MG). Está inserido entre os municípios de Ouro Preto e Mariana, a sudeste do Quadrilátero Ferrífero, no Estado de Minas Gerais. O clima da região é do tipo Cwa e Cwb, conforme a classificação de Köeppen, com temperatura média de $21^{\circ} \mathrm{C}$, máxima de $33^{\circ} \mathrm{C}$ e mínima de $4^{\circ} \mathrm{C}$ (SEMAD/IEF/ PROMATA 2007). A pluviosidade média anual varia de $1.100 \mathrm{~mm}$ a $1.800 \mathrm{~mm}$, com pico entre dezembro e janeiro e uma estação seca bem definida entre os meses de abril a setembro (SEMAD/IEF/PROMATA 2007) (Fig. 1). A área amostrada é um fragmento de floresta paludosa, de aproximadamente $80 \times 35 \mathrm{~m}^{2}$, a $1.400 \mathrm{~m}$ de altitude, localizado entre a margem direita do Córrego do Manso e uma estrada de terra de circulação interna do Parque. Foi estabelecido um transecto de $60 \mathrm{~m}$, localizado a $17,5 \mathrm{~m}$ da margem do córrego e em sentido paralelo ao seu curso. Ao longo do transecto foram definidos cinco pontos amostrais eqüidistantes entre si (P1 a P5), com a finalidade de amostrar a umidade do solo e mensurar a decomposição da serapilheira foliar. Em seguida, foram alocadas parcelas de $10 \times 10 \mathrm{~m}$ para a coleta de serapilheira, sendo uma parcela à esquerda e uma à direita de cada ponto amostral.

\section{Caracterização do solo}

Amostras compostas de solo (0-10 cm de profundidade) foram coletadas em cada um dos cinco pontos amostrais do transecto no início da estação seca (28/05/2007), auge (28/07/2007) e final desta estação (28/09/2007). Cada amostra foi composta por três sub-amostras coletadas no ponto central e a $3 \mathrm{~m}$ à esquerda e à direita deste ponto. As coletas foram feitas utilizando-se um tubo de PVC com $4 \mathrm{~cm}$ de diâmetro. O conteúdo de água, expresso como a diferença em porcentagem entre o peso úmido e o peso seco de cada amostra, foi calculado pelo método gravimétrico (Embrapa 1997), após secagem em estufa a $60^{\circ} \mathrm{C}$ durante $72 \mathrm{~h}$. As concentrações de P-disponível e o $\mathrm{pH}$ em $\mathrm{H}_{2} \mathrm{O}$ foram analisados apenas para as amostras de 28/05/2007, de acordo com metodologia proposta por Embrapa (1997).

\section{Produção de serapilheira}

Para as coletas de serapilheira foi instalado no centro de cada parcela um coletor de $0,25 \mathrm{~m}^{2}$ com tela de náilon (malha de $1 \mathrm{~mm}^{2}$ ) a aproximadamente $20 \mathrm{~cm}$ do solo, de acordo com a metodologia proposta por Moraes et al. (1999). Os coletores foram alocados na área no dia 14/09/2006. O material depositado em cada um dos coletores foi coletado mensalmente entre 14/10/2006 e 15/09/2007, e separado nas frações de folhas e miscelânea (frutos, sementes, galhos e cascas). Foram descartados os galhos com mais de $2 \mathrm{~cm}$ de diâmetro (Arato et al. 2003). Após a triagem, o material foi seco em estufa a $60{ }^{\circ} \mathrm{C}$ por $48 \mathrm{~h}$ e o peso seco obtido em balança analítica com precisão de três casas decimais e expresso em $t$ ha $^{-1}$.

A fim de se verificar o efeito da sazonalidade sobre a qualidade nutricional da serapilheira foliar, foram se- 


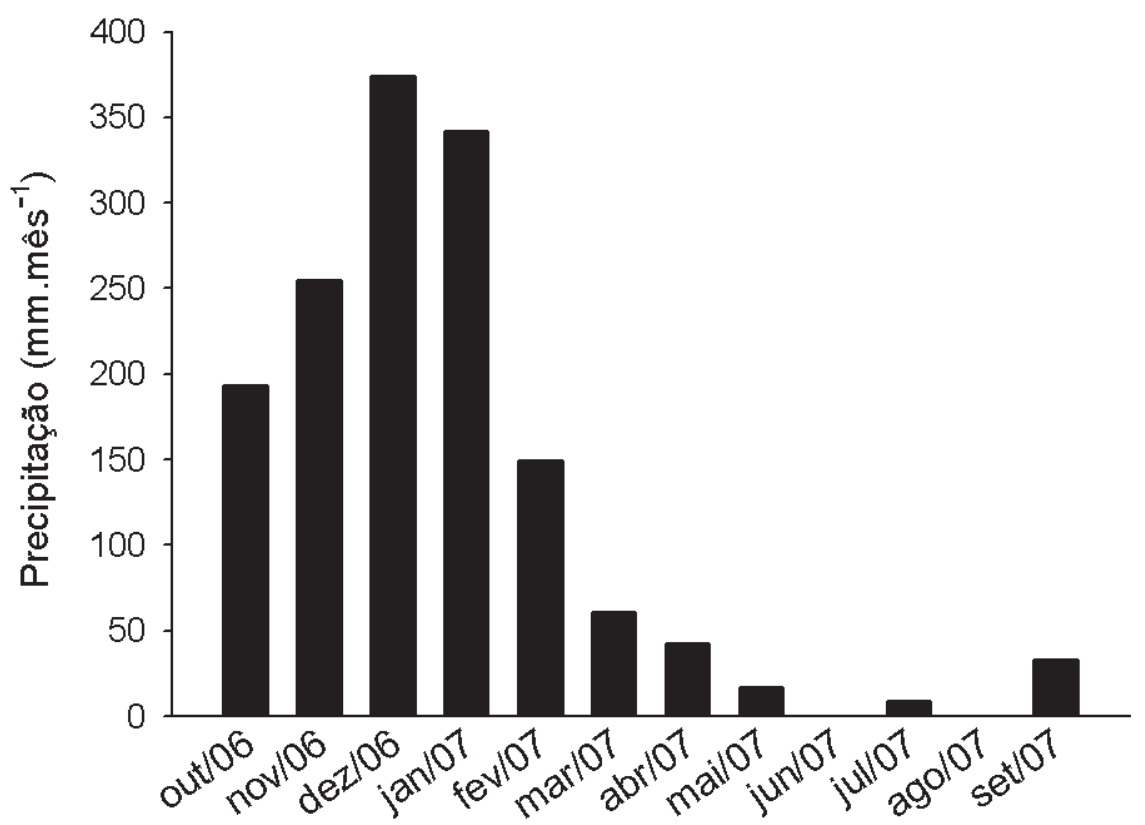

Figura 1. Precipitação mensal nas proximidades do Parque Estadual do Itacolomi, MG, Brasil, entre outubro de 2006 e setembro de 2007. Dados cedidos pela Novelis do Brasil Ltda, Unidade Ouro Preto, Estação Saramenha. O período entre os meses de outubro a março corresponde à estação chuvosa na região.

paradas amostras compostas depositadas nos coletores de serapilheira, referentes à transição entre as estações seca e chuvosa $(14 / 10 / 2006 ; n=3)$, auge da chuvosa $(15 / 01 / 2007 ; n=3)$, transição entre as estações chuvosa e seca $(14 / 05 / 2007, \mathrm{n}=3)$ e final da estação seca (15/09/2007; $\mathrm{n}=3$ ). O material amostrado foi enviado ao Laboratório de Análise Foliar da Universidade Federal de Viçosa (UFV) para análise de N, P, K e C-total. O N foi determinado pelo método Kjeldahl, descrito por Bataglia et al. (1983); o P por colorimetria, através do método do complexo fosfomolíbdico, reduzido com vitamina $\mathrm{C}$, modificado por Braga \& Defelipo (1974) e o K foi dosado em fotômetro de chama (Bataglia et al. 1983).

A transferência média mensal de $\mathrm{N}, \mathrm{P}, \mathrm{K}$ e $\mathrm{C}$ da vegetação para a serapilheira foliar durante os meses de outubro/2006, janeiro/2007, maio/2007 e setembro/2007 foi calculada como o produto entre a produção média de serapilheira foliar de cada período analisado e a respectiva concentração de nutrientes (Vital et al. 2004). A quantidade de $\mathrm{N}, \mathrm{P}, \mathrm{K}$ e C transferida da vegetação para a serapilheira foliar durante um ano foi estimada calculando-se o produto entre a produção média anual de serapilheira foliar e a média entre as concentrações de nutrientes nos meses de outubro/2006, janeiro/2007, maio/2007 e setembro/2007.

\section{Decomposição da serapilheira foliar}

Para a estimativa da decomposição da serapilheira foliar foram utilizadas cinco bolsas de decomposição de
$15 \times 15 \mathrm{~cm}$, confeccionadas em tela de náilon (malha de $1 \mathrm{~mm}^{2}$ ), cada uma contendo $5 \mathrm{~g}$ de folhas recém-caídas, coletadas em maio de 2007 e previamente secas em estufa a $60^{\circ} \mathrm{C}$ durante $72 \mathrm{~h}$ (Graça et al. 2005). As bolsas de decomposição foram dispostas sobre o solo no dia 28/05/2007 em cada um dos pontos amostrais do transecto, onde permaneceram por 170 dias até 14/11/2007. Após este período, as amostras foram secas em uma estufa a $60^{\circ} \mathrm{C}$ por 48 horas e pesadas em balança analítica com precisão de três casas decimais. A decomposição da serapilheira foliar foi expressa em porcentagem de massa perdida $(\% \mathrm{MP})$ em relação à massa seca original de cada bolsa de decomposição.

\section{Análises estatísticas}

A distribuição dos dados foi analisada pelo teste de Kolmogorov-Smirnov a fim de se verificar o ajuste à curva normal. Variações sazonais no conteúdo de água no solo, produção, qualidade nutricional e taxa de decomposição da serapilheira foram testadas através de ANOVA unifatorial (teste F), utilizando e as médias separadas por LSD. As variações espaciais foram testadas utilizando-se ANOVA (teste F) e teste $\mathrm{T}$ de Student. A relação entre o conteúdo de água no solo e a porcentagem de massa perdida durante a decomposição da serapilheira foliar foi testada através do teste de correlação de Pearson e regressão linear. O índice de significância $(\alpha)$ adotado foi 0,05 . Foi utilizado o pacote estatístico SPSS versão 15.0. 


\section{Resultados e discussão}

\section{Caracterização do solo}

O conteúdo médio de água no solo $(0-10 \mathrm{~cm})$ da área estudada se manteve relativamente constante durante a estação seca, com média de $53,4 \pm 13,6 \%$. De acordo com os valores observados, a área estudada foi dividida em duas regiões com diferenças significativas em relação ao conteúdo de água no solo (Tab. 1). A Zona 1 (mais úmida / pontos amostrais $\mathrm{P} 1, \mathrm{P} 2$ e $\mathrm{P} 3$ ), apresentou um conteúdo médio de água relativamente constante ao longo do ano e $65 \%$ mais alto em relação à Zona 2 (pontos amostrais $\mathrm{P} 4 \mathrm{e}$ P5) que, por sua vez, apresentou uma queda significativa no conteúdo médio de água do solo entre o início (maio/2007) e o final (set/2007) da estação seca (Tab. 1).

De acordo com a classificação proposta por Junior et al. (2003), o solo da floresta paludosa pode ser considerado como "saturado" na Zona 1 ( $\geq 61 \%)$ e "semi saturado" na Zona 2 ( $\geq 35 \%$ e $\leq 60 \%)$. Outro estudo realizado em dois fragmentos de floresta paludosa no Parque Estadual do Itacolomi encontrou forte relação entre a diferenciação espacial no conteúdo de água do solo superficial e a riqueza de espécies vegetais nesses ecossistemas, mesmo em escala de metros a dezenas de metros (Pedreira 2008). Variações espaciais na riqueza e distribuição de espécies, por sua vez, podem influenciar a ciclagem de nutrientes, devido à distinta capacidade de absorção e devolução de nutrientes das diferentes espécies vegetais. A saturação hídrica do solo exerce papel significativo não somente sobre a dinâmica de produção de serapilheira, mas também sobre sua taxa de decomposição, que tende a ser reduzida devido aos baixos teores de oxigênio do solo (Pagano \& Durigan 2000; Hobbie \& Vitousek 2000).

$\mathrm{O} \mathrm{pH}$ do solo $(0-10 \mathrm{~cm})$ da floresta paludosa variou entre 4,3 e 5,0, indicando uma acidez relativamente alta, padrão recorrente em diversas florestas paludosas do sudeste do Brasil (Torres et al. 1994; Toniato et al. 1998). A disponibilidade de $\mathrm{P}$ no solo pode ser considerada entre baixa e moderada $\left(1,2\right.$ a $\left.4,1 \mathrm{mg} \mathrm{dm}^{-3}\right)$ e pode estar relacionada à elevada acidez do solo. Segundo Chapin et al. (2002), sob condições de baixo pH, a disponibilidade de $\mathrm{P}$ no solo tende a diminuir consideravelmente, devido à formação de fosfatos inorgânicos insolúveis, resultando em um crescimento fortemente limitado por P na maioria dos ecossistemas florestais tropicais. Em um estudo realizado em solos inundáveis de veredas em Minas Gerais, Ramos et al. (2006) observaram teores de P disponível no solo entre 2 e $5 \mathrm{mg} \mathrm{dm}^{-3}$, valores dentro da faixa observada no presente estudo.

\section{Produção de Serapilheira}

A deposição anual média de serapilheira na floresta paludosa foi igual a $5,68 \mathrm{tha}^{-1}$, sendo a fração foliar responsável por $57 \%$ da produção total $\left(3,22 \mathrm{t} \mathrm{ha}^{-1}\right)$. Tais valores encontram-se dentro dos limites mais baixos da faixa observada em ecossistemas florestais tropicais, que varia entre $5,35 \mathrm{t} \mathrm{ha}^{-1}$ (Pagano \& Durigan 2000) e 12,22 $\mathrm{t} \mathrm{ha}^{-1}$ (Hora et al. 2008), com a fração foliar atingindo até $80 \%$ da produção total. Em uma meta análise sobre processos ligados à ciclagem de nutrientes em uma grande variedade de florestas tropicais úmidas da América do Sul, América Central, Ásia e Oceania, foram observados valores médios de produção de serapilheira com variação entre $10,4 \mathrm{t} \mathrm{ha}^{-1} \mathrm{em}$ florestas sobre solos moderadamente férteis, $8,8 \mathrm{t} \mathrm{ha}^{-1}$ para solos de baixa fertilidade (oxisolos) e 6,4 $\mathrm{tha}^{-1} \mathrm{em}$ florestas tropicais úmidas de altitude (Vitousek \& Sanford Jr. 1986).

Diversos estudos indicam uma correlação negativa entre disponibilidade hídrica e produção de serapilheira (Vitousek 1984), demonstrando que ecossistemas sujeitos à condição de saturação hídrica e alagamentos, mesmo que temporários, tendem a apresentar menor deposição de serapilheira, especialmente da fração foliar. Esta pode ser uma importante estratégia capaz de minimizar as perdas de nutrientes devido ao escoamento da água sobre o solo em direção aos cursos d'água (Pagano \& Durigan 2000; Pinto et al. 2008). Portanto, a baixa deposição de serapilheira na floresta paludosa poderia estar relacionada à ausência de déficit hídrico, uma vez que a maior parte das amostras de solo apresentou conteúdo de água entre saturado e semisaturado, mesmo durante os períodos mais secos do ano.

Não houve diferenças significativas na produção de serapilheira total (ST) ou foliar (SF) entre a zona 1 (ST=0,49 $\left.\pm 0,34 \mathrm{tha}^{-1} / \mathrm{SF}=0,26 \pm 0,19 \mathrm{tha}^{-1}\right)$ e a zona $2(\mathrm{ST}=0,44 \pm$ $\left.0,27 \mathrm{t} \mathrm{ha}^{-1} / \mathrm{SF}=0,28 \pm 0,16 \mathrm{tha}^{-1}\right)$, indicando que mesmo os solos da zona semi-saturada (zona 2) possuem conteúdo de água superior aos encontrados em solos não hidromór-

Tabela 1. Conteúdo médio de água $(\%)$ no solo $(0-10 \mathrm{~cm})$ nas duas zonas definidas dentro do fragmento de floresta paludosa estudado, durante o início (maio/2007, $\mathrm{n}=5$ ), meio (julho/2007, n=5) e final (setembro/2007, $\mathrm{n}=5$ ) da estação seca. Média seca = média entre os três períodos analisados, correspondente à estação seca $(\mathrm{n}=15)$. Letras diferentes indicam diferenças entre as médias em uma mesma coluna (ANOVA; $\mathrm{p}<0,05)$. Asteriscos indicam diferenças entre as médias em uma mesma linha (Teste $\mathrm{t}, \mathrm{P}<0,05)$.

\begin{tabular}{|c|c|c|}
\hline & Zona 1 (\%) & Zona $2(\%)$ \\
\hline maio/2007 & $63,8 \pm 2,9 a$ & $48,6 \pm 1,7 \mathrm{a}$ \\
\hline julho/2007 & $63,9 \pm 1,0 \mathrm{a}$ & $33,6 \pm 1,9 b$ \\
\hline setembro/2007 & $62,6 \pm 2,8 \mathrm{a}$ & $33,2 \pm 2,4 b$ \\
\hline média estação seca & $63,4 \pm 2,2^{*}$ & $38,4 \pm 8,0$ * \\
\hline
\end{tabular}


ficos excluindo, portanto, a possibilidade de alterações na produção de serapilheira em resposta ao desenvolvimento de déficit hídrico.

A deposição de serapilheira seguiu um padrão sazonal, com pico de produção durante a transição entre as estações seca e chuvosa (setembro), especialmente para a fração foliar (Fig. 2). Um segundo pico de produção foi ainda observado durante o mês de janeiro, auge da estação chuvosa, fortemente influenciado pela fração miscelânea, em decorrência da alta produção de frutos e ritidomas de Myrcia laruotteana, espécie dominante no local (Fig. 2).

Esse padrão é comum a diversos ecossistemas florestais sazonais do sudeste brasileiro (Haase 1999; Martins \& Rodrigues 1999; Villela \& Proctor 1999; Pagano \& Durigan 2000; Cianciaruso et al. 2006). Entretanto, diversos autores relacionam as maiores taxas de produção de serapilheira durante o final da estação seca ao déficit hídrico provocado por um longo período de estiagem (Vitousek 1984; Pagano \& Durigan 2000; Pinto et al. 2008). Como a vegetação da floresta paludosa aparentemente não experimenta condição de déficit hídrico, mesmo após longos períodos de seca, a produção de serapilheira nesses ecossistemas deve estar condicionada a outros fatores, como a ocorrência de dias mais curtos, com menor disponibilidade de luz (Pagano \& Durigan 2000; Taiz \& Zeiger 2004) e baixas temperaturas durante o inverno (Larcher 2000; Pinto et al. 2008), em detrimento da disponibilidade hídrica. Os valores relativamente altos e constantes para a deposição de serapilheira durante a estação chuvosa (outubro a março) podem estar relacionados à ocorrência de fortes temporais e ventos durante esse período (Martins \& Rodrigues 1999), enquanto a queda na produção durante o início da estação seca (maio a julho) pode estar relacionada ao fim das chuvas.

A Tab. 2 apresenta as concentrações médias de N, P, K e C, além das relações entre C:N e N:P observadas na serapilheira foliar produzida na floresta paludosa durante os períodos analisados. De maneira geral, os teores de N, P e $\mathrm{K}$ encontram-se dentro dos limites mais baixos observados para florestas tropicais brasileiras, que variam entre 10,7 e 24,4 $\mathrm{g} \mathrm{kg}^{-1}$ para N (Villela \& Proctor 1999; Pinto et al. 2009), 0,27 e 2,08 $\mathrm{g} \mathrm{kg}^{-1}$ para P (Vasconcelos \& Luizão 2004; Haase 1999) e 2,93 a 11,8 $\mathrm{g} \mathrm{kg}^{-1}$ para K (Pinto et. al 2009; König et al. 2002). Pagano \& Durigan (2000) observaram em florestas inundáveis (matas de galeria) do interior de São Paulo valores relativamente mais altos para $\mathrm{N}\left(15,2\right.$ a $\left.21,5 \mathrm{~g} \mathrm{~kg}^{-1}\right)$ e K (4,2 a 5,5 $\left.\mathrm{g} \mathrm{kg}^{-1}\right)$, enquanto os teores de P $(0,6$ a $0,7 \mathrm{~g}$ $\mathrm{kg}^{-1}$ ) encontram-se próximos aos observados no presente estudo. Entretanto, em um estudo realizado em florestas inundáveis perenes no centro-oeste brasileiro, Haase (1999) observou valores até $200 \%$ mais altos em relação aos valores encontrados na floresta paludosa.

As concentrações de $\mathrm{N}$ e P na serapilheira foliar (Tab. 2) apresentaram os maiores valores durante o início da estação seca (maio), sendo que as concentrações de $\mathrm{P}$ apresentaram um aumento gradual ao longo da estação chuvosa, indicando que, nos períodos mais secos, a vegetação da floresta paludosa apresenta uma maior proficiência de retranslocação de P durante o processo

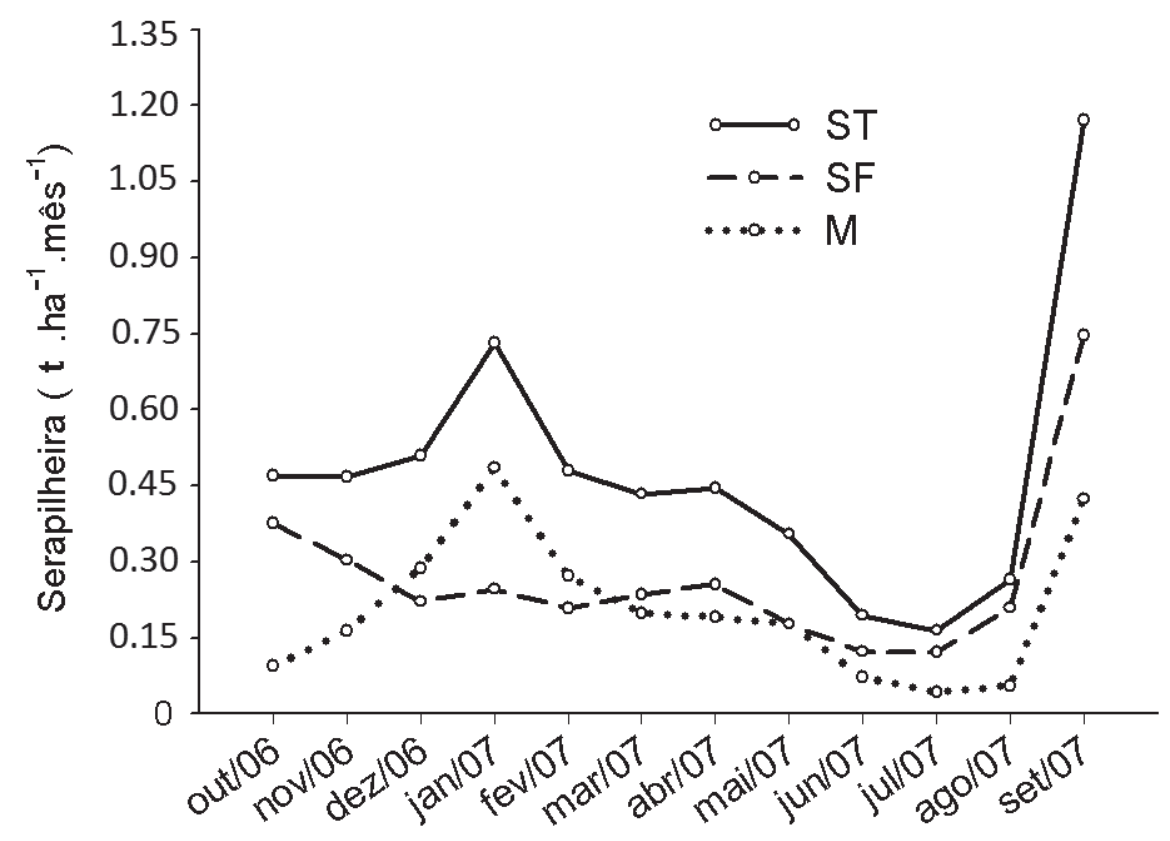

Figura 2. Produção média mensal de serapilheira total $(S T / n=10)$, serapilheira foliar $(S F / n=10)$ e miscelânea (M / n=10) (em t ha-1 mês ${ }^{-1}$ ) em um fragmento de floresta paludosa no Parque Estadual do Itacolomi, MG, Brazil, entre outubro de 2006 e setembro de 2007. Estação chuvosa: outubro a março/ estação seca: abril a setembro. 
de senescência foliar (Killingbeck 1996). Não houve qualquer variação sazonal significativa nos teores de $\mathrm{Ke} C$ na serapilheira foliar (Tab. 2). Também não houve quaisquer variações espaciais nas concentrações de nutrientes da serapilheira foliar $\left(\mathrm{N}_{\text {Zona1 }}=12,8 \mathrm{~g} \mathrm{~kg}^{-1} \mathrm{~N}_{\text {Zona2 }}=12,2 \mathrm{~g} \mathrm{~kg}^{-1}\right.$ / $\mathrm{P}_{\text {Zonal }}=0,62 \mathrm{~g} \mathrm{~kg}^{-1} \mathrm{P}_{\text {Zona2 }}=0,60 \mathrm{~g} \mathrm{~kg}^{-1} / \mathrm{K}_{\text {Zona1 }}=3,1 \mathrm{~g} \mathrm{~kg}^{-1}$ $\mathrm{K}_{\text {Zona } 2}=2,9 \mathrm{~g} \mathrm{~kg}^{-1}$ ), sugerindo que a diferença na umidade do solo superficial entre as zonas 1 (solo saturado) e 2 (solo semi-saturado) não foi suficiente para ocasionar variações na qualidade nutricional da serapilheira foliar depositada na floresta paludosa.

A razão C:N (Tab. 2) reflete a variação sazonal do $\mathrm{N}$ na serapilheira foliar, uma vez que os teores de C se mantiveram relativamente constantes durante os períodos analisados. Além disso, a relação entre $\mathrm{C}$ e $\mathrm{N}$ pode ser utilizada como indicador da qualidade nutricional da serapilheira, sendo que valores maiores que 25 indicam que o material foliar apresenta uma baixa proporção de N, limitando assim o processo de decomposição (Luizão et al. 2004). Os valores observados neste estudo (Tab. 2) indicam que a baixa qualidade nutricional da serapilheira pode ser um importante fator limitante da decomposição do material foliar depositado na floresta paludosa, além de refletir uma maior eficiência por parte da vegetação na conservação de $\mathrm{N}$ em seus tecidos vivos em relação a outros ecossistemas florestais tropicais.

A razão N:P em tecidos vegetais tem sido amplamente utilizada para determinar se uma espécie ou um ecossistema é limitado por P ou N (Bedford et al. 1999; Aerts \& Chapin 2000; Güsewell 2004; Kozovits et al. 2007). Segundo Aerts \& Chapin (2000), valores de N:P $<14$ indicam limitação por nitrogênio, $\mathrm{N}: \mathrm{P}>16$ indicam limitação por fósforo e valores entre 14 e 16 indicam co-limitação por N e P. A razão N:P da serapilheira foliar na floresta paludosa estudada (Tab. 2) aponta uma forte limitação por fósforo durante todo o ano, padrão comum a diversos ecossistemas florestais tropicais, que tendem a apresentar forte limitação por $\mathrm{P}$ devido às baixas concentrações deste elemento nos solos antigos e altamente intemperizados dessas regiões (Aerts \& Chapin 2000; Chapin et al. 2002; Davidson \& Howarth 2007). A razão N:P na serapilheira foliar está diretamente ligada à taxa de retranslocação de $\mathrm{N}$ e $\mathrm{P}$ nos tecidos foliares durante a abscisão foliar, mecanismo que se acredita ter evoluído de maneira a conservar nutrientes em ambientes com fortes limitações nutricionais (Killingbeck 1996).

Güsewell (2004) relata a influência das atividades humanas sobre a regulação de processos ligados à ciclagem de nutrientes em diversos ecossistemas. Uma associação entre fatores como a queima de combustíveis fósseis e o uso de fertilizantes agrícolas praticamente dobraram a taxa de entrada de $\mathrm{N}$ na biosfera terrestre em formas disponíveis à maioria dos seres vivos. Por outro lado, as perdas anuais de $\mathrm{P}$ em ecossistemas terrestres devido a atividades humanas são cada vez maiores com o passar do tempo. Como resultado, esses ecossistemas têm se tornado cada vez mais limitados por $\mathrm{P}$.

As quantidades de nutrientes transferidos da vegetação para a serapilheira foliar (Tab. 3) encontram-se abaixo dos valores observados em grande parte dos ecossistemas florestais brasileiros, principalmente em razão da relativamente baixa deposição anual de serapilheira na floresta paludosa e de seus baixos teores de nutrientes. Em uma floresta secundária no município de Seropédica, RJ, Fernandes et al. (2006) observaram uma transferência de $149,8 \mathrm{~kg}(\mathrm{~N})$ $\mathrm{ha}^{-1}, 3,00 \mathrm{~kg}(\mathrm{P}) \mathrm{ha}^{-1}$ e $16,3 \mathrm{~kg}(\mathrm{~K}) \mathrm{ha}^{-1}$. Moraes et al. (1999) observaram um fluxo anual de 71,9 $\mathrm{kg}(\mathrm{N}) \mathrm{ha}^{-1}, 2,3 \mathrm{~kg}(\mathrm{P})$ $\mathrm{ha}^{-1}$ e $12,6 \mathrm{~kg}(\mathrm{~K}) \mathrm{ha}^{-1}$ em um trecho de floresta úmida na Ilha do Cardoso, SP. Montagnini \& Jordan (2005) propuseram um valor médio para o fluxo anual de nutrientes, através da serapilheira, em torno de $42,0 \mathrm{~kg} \mathrm{ha}^{-1}$ para $\mathrm{N}$ e 2,6 kg ha-1 para $\mathrm{P}$ em solos de fertilidade extremamente baixa. A variação sazonal na transferência de nutrientes da vegetação para a serapilheira foliar se mostrou diretamente dependente da deposição de serapilheira em detrimento de sua qualidade nutricional, uma vez que o maior fluxo de nutrientes ocorreu em setembro, final da estação seca, mesmo com os menores teores de nutrientes na serapilheira foliar durante esse período.

\section{Decomposição da Serapilheira Foliar}

A serapilheira foliar depositada durante o mês de maio de 2007 na floresta paludosa apresentou uma perda de massa de $36 \%$ em relação à massa seca original durante um período de 170 dias ao longo da estação seca. Tais resultados

Tabela 2. Concentrações de N, P, K, C $\left(\mathrm{g} \mathrm{kg}^{-1}\right)$ e relações entre carbono e nitrogênio (C:N) e nitrogênio e fósforo (N:P) na serapilheira foliar em um trecho de floresta paludosa no Parque Estadual do Itacolomi, MG, Brasil, durante os meses de outubro de 2006 ( $n=3$ ), janeiro ( $n=3$ ), maio ( $n=3$ ) e setembro ( $n=3$ ) de 2007. Média total $=$ média entre os quatro períodos analisados $(\mathrm{n}=12)$. Letras diferentes indicam diferenças significativas entre as médias em uma mesma coluna $($ ANOVA; $<<0,05)$.

\begin{tabular}{|c|c|c|c|c|c|c|}
\hline & $\mathrm{N}\left(\mathrm{g} \cdot \mathrm{kg}^{-1}\right)$ & $\mathrm{P}\left(\mathrm{g} \cdot \mathrm{kg}^{-1}\right)$ & $\mathrm{K}\left(\mathrm{g} \cdot \mathrm{kg}^{-1}\right)$ & $\mathrm{C}\left(\mathrm{g} \cdot \mathrm{kg}^{-1}\right)$ & $\mathrm{C}: \mathrm{N}$ & $\mathrm{N}: \mathrm{P}$ \\
\hline outubro/2006 & $12,6 \pm 0,5 \mathrm{ab}$ & $0,593 \pm 0,01 \mathrm{ab}$ & $2,92 \pm 0,2 \mathrm{a}$ & $549,2 \pm 1,5 \mathrm{a}$ & $44 \pm 2 \mathrm{ab}$ & $21 \pm 1 b$ \\
\hline janeiro/2007 & $12,1 \pm 1,0 \mathrm{a}$ & $0,633 \pm 0,04 b c$ & $3,37 \pm 0,8 a$ & $548,3 \pm 3,7 \mathrm{a}$ & $45 \pm 3 b$ & $19 \pm 1 \mathrm{a}$ \\
\hline maio/2007 & $13,8 \pm 0,8 b$ & $0,657 \pm 0,02 c$ & $3,17 \pm 0,7 \mathrm{a}$ & $548,0 \pm 4,3 \mathrm{a}$ & $40 \pm 2 \mathrm{a}$ & $21 \pm 1 b$ \\
\hline setembro/2007 & $12,0 \pm 0,1 \mathrm{a}$ & $0,579 \pm 0,018 a$ & $2,82 \pm 0,1 \mathrm{a}$ & $550,5 \pm 1,1 \mathrm{a}$ & $46 \pm 1 b$ & $21 \pm 1 b$ \\
\hline média anual & $12,6 \pm 1,0$ & $0,616 \pm 0,04$ & $3,07 \pm 0,5$ & $549,0 \pm 2,7$ & $44 \pm 3$ & $21 \pm 1$ \\
\hline
\end{tabular}


Tabela 3. Transferência de N, P, Ke C $\left(\mathrm{kg} \mathrm{ha}^{-1}\right)$ da vegetação para a serapilheira foliar em um fragmento de floresta paludosa no Parque Estadual do Itacolomi, MG, Brasil, durante os meses de outubro de $2006(n=3)$, janeiro $(n=3)$, maio $(n=3)$ e setembro $(n=3)$ de 2007. Estimativa anual= calculada a partir do produto entre a produção anual média de serapilheira foliar e a concentração média dos nutrientes relativa aos quatro períodos analisados. Letras diferentes indicam diferenças significativas entre as médias em uma mesma coluna (ANOVA, $\mathrm{P}<0,05$ )

\begin{tabular}{|c|c|c|c|c|}
\hline & $\mathrm{N}\left(\mathrm{kg} \mathrm{ha}^{-1}\right)$ & $\mathrm{P}\left(\mathrm{kg} \mathrm{ha}^{-1}\right)$ & $\mathrm{K}\left(\mathrm{kg} \mathrm{ha}^{-1}\right)$ & $\mathrm{C}\left(\mathrm{kg} \mathrm{ha}^{-1}\right)$ \\
\hline outubro/2006 & $4,7 \pm 0,2 \mathrm{c}$ & $0,223 \pm 0,005 c$ & $1,10 \pm 0,07 \mathrm{c}$ & $206,5 \pm 0,6 c$ \\
\hline janeiro/2007 & $3,0 \pm 0,2 b$ & $0,156 \pm 0,011 b$ & $0,83 \pm 0,21 b$ & $135,0 \pm 0,9 b$ \\
\hline maio/2007 & $2,4 \pm 0,1 \mathrm{a}$ & $0,117 \pm 0,004 a$ & $0,56 \pm 0,13 \mathrm{a}$ & $97,2 \pm 0,8 \mathrm{a}$ \\
\hline setembro/2007 & $9,0 \pm 0,1 \mathrm{~d}$ & $0,432 \pm 0,014 \mathrm{~d}$ & $2,11 \pm 0,09 \mathrm{~d}$ & $411,0 \pm 0,8 \mathrm{~d}$ \\
\hline Estimativa Anual & 40,7 & 1,98 & 9,89 & 1769,3 \\
\hline
\end{tabular}

indicam uma decomposição relativamente lenta quando comparada a outros ecossistemas florestais tropicais.

Cleveland et al. (2006) observaram em uma floresta tropical úmida no sudeste da Costa Rica uma perda de massa da serapilheira foliar superior a $50 \%$ em um período de 120 dias. Em uma área de floresta secundária no município de Seropédica, RJ, a perda de massa após 120 dias de decomposição da serapilheira foliar foi superior a $40 \%$ (Fernandes et al. 2006). Em um estudo realizado com 10 espécies nativas da mata atlântica, Arato (2006) considerou como espécies de decomposição lenta aquelas cuja serapilheira foliar apresentou perda de massa inferior a 50\% durante 180 dias. No processo de decomposição da serapilheira foliar em uma floresta ombrófila densa na Reserva Natural de Salto Morato, PR foi observada uma perda de massa de 31\% após 180 dias (Scheer 2008).

Diversos estudos mostram que a qualidade nutricional da serapilheira desempenha um importante papel na regulação do processo de decomposição em diversos ecossistemas florestais (Hobbie \& Vitousek 2000; Villela \& Proctor 2002; Cleveland et al. 2006; Kozovits et al. 2007). Nitrogênio e fósforo orgânicos são os elementos mais importantes para o crescimento de microrganismos envolvidos no processo de decomposição da matéria orgânica. Assim, baixas concentrações desses nutrientes podem limitar o crescimento dos microrganismos, retardando consideravelmente a decomposição do material (Chapin et al. 2002). Hobbie \& Vitousek (2000) observaram forte limitação do processo de decomposição pelas concentrações de P, tanto no solo quanto na serapilheira foliar, em uma floresta tropical montana no Havaí. Portanto, a baixa qualidade nutricional da serapilheira foliar depositada parece ser um importante fator limitante do processo de decomposição da serapilheira foliar na floresta paludosa.

Outro fator que pode ser responsável pela decomposição relativamente lenta na floresta paludosa em relação a outros ecossistemas florestais tropicais são as baixas temperaturas registradas na região, podendo ocorrer, durante o inverno, temperaturas próximas a $0^{\circ} \mathrm{C}$ (IEF/SEMAD/PROMATA 2007). Segundo Chapin et al. (2002), a temperatura afeta diretamente a decomposição da matéria orgânica, exercendo forte influência sobre a atividade microbiana no solo, além de, indiretamente alterar condições microclimáticas de umidade.
A porcentagem de massa perdida durante a decomposição mostrou-se negativamente correlacionada com o conteúdo de água no solo $\left(\mathrm{R}^{2}=0,9205 ; \mathrm{p}=0,0098\right)$ (Fig. 3). $\mathrm{Na}$ Zona 1, onde foi registrada a maior porcentagem de água no solo, observou-se uma perda de massa de 29,2\%, valor significativamente menor do que o registrado na Zona 2 (46,2\%), onde o solo apresentou um menor conteúdo de água. Em grande parte dos ecossistemas sazonais existe uma relação positiva entre o conteúdo de água no solo superficial e a decomposição da serapilheira, ou seja, a decomposição é mais rápida em períodos de chuva e maior umidade no solo, quando as condições para o desenvolvimento de microrganismos decompositores são mais favoráveis e as chuvas carregam por lixiviação íons inorgânicos e alguns nutrientes, facilitando o processo de decomposição da serapilheira (Montagnini \& Jordan 2005; Kozovits et al. 2007). Entretanto, a condição de saturação do solo das florestas paludosas e os alagamentos constantes devido ao afloramento do lençol freático podem levar a uma situação de hipoxia do solo, reduzindo a ação de microrganismos decompositores e o desenvolvimento e atividade de raízes finas, que deixam de excretar enzimas e substâncias que podem facilitar a decomposição (Berg \& McClaugherty 2007). Assim, apesar da decomposição da serapilheira ser favorecida pelo aumento da umidade do solo na maioria dos ecossistemas sazonais da região tropical, aparentemente, existe um limite de saturação do solo a partir do qual o aumento na umidade passa a exercer um efeito semelhante ao ocasionado pelo déficit hídrico, reduzindo a velocidade com que a matéria orgânica depositada sobre o solo é decomposta. De fato, a porcentagem de massa perdida durante a decomposição observada no presente estudo assemelha-se aos valores observados em ecossistemas sujeitos a constantes situações de déficit hídrico, como ambientes de cerrado (Cianciaruso et al. 2006).

\section{Conclusão}

A condição de saturação hídrica do solo parece ser fator determinante da baixa produção e decomposição de serapilheira foliar na área estudada quando comparadas a valores encontrados em outros ecossistemas florestais tropicais. A 


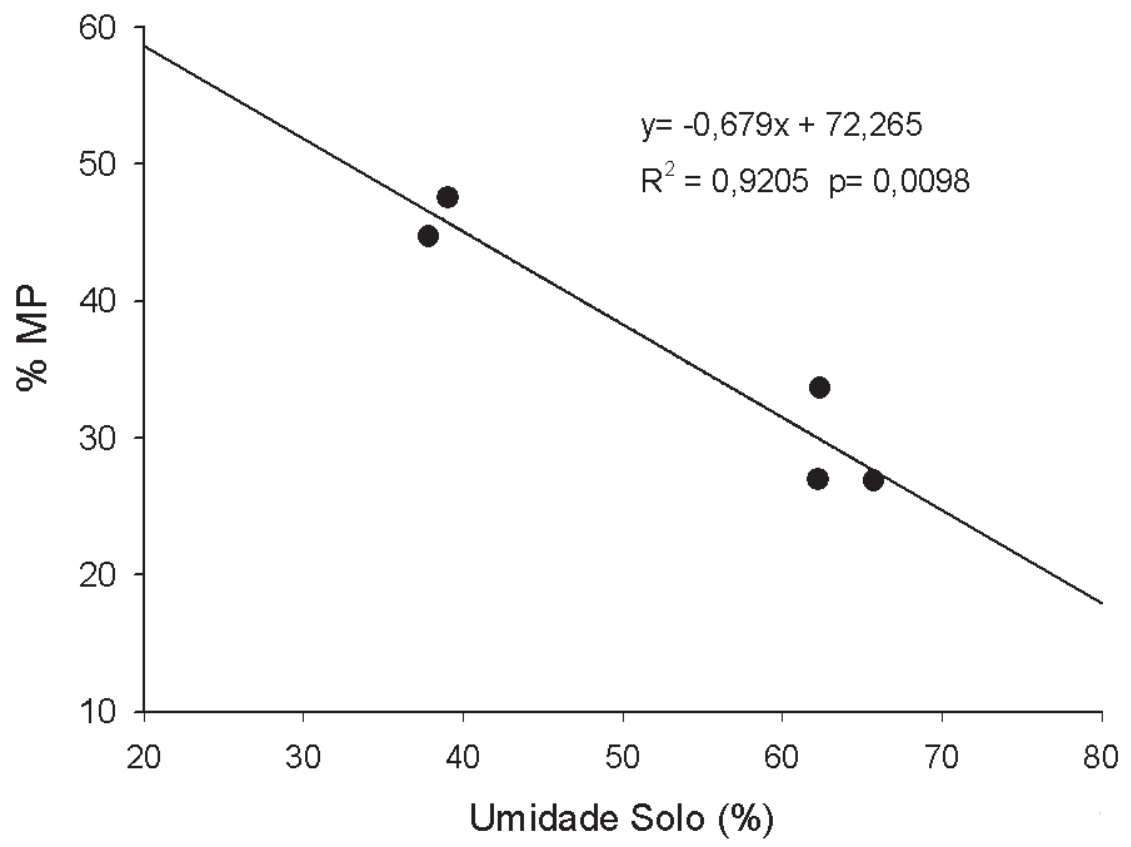

Figura 3. Correlação entre a porcentagem de massa seca perdida $(\% \mathrm{MP} / \mathrm{n}=5)$ pela serapilheira foliar após 170 dias de decomposição in situ e o conteúdo de água no solo superficial (\% água/n=5) de um fragmento de floresta paludosa no Parque Estadual do Itacolomi, MG, Brasil.

relação inversa entre o conteúdo de água no solo e a perda de massa durante a decomposição da serapilheira, encontrada no presente trabalho, sugere que pelo menos parte dos processos envolvidos na ciclagem de nutrientes em sistemas florestais sujeitos à inundação é controlada pelos níveis de saturação de água no solo, o que pode variar de maneira significativa entre zonas separadas apenas por poucos metros na área de estudo. Somando-se a isso, a baixa qualidade nutricional da serapilheira foliar e as baixas temperaturas de inverno também devem limitar o processo de decomposição, diminuindo consideravelmente a velocidade com que os nutrientes na serapilheira foliar retornam ao solo e se tornam novamente disponíveis para a vegetação na floresta paludosa estudada. Por outro lado, a sazonalidade na deposição de serapilheira não pôde ser explicada pela disponibilidade de água no solo, que se manteve constante e relativamente alta durante todo o período analisado, indicando que outros fatores ambientais devam exercer papéis mais relevantes sobre os padrões fenológicos da floresta paludosa de altitude.

\section{Referências bibliográficas}

Aerts, R. \& Chapin, F. 2000. The mineral nutrition of wild plants revisited: a re-evaluation of processes and patterns. Advanced Ecolological Research 30: 1-67.

Arato, H.D. 2006. Caracterização química e decomposição de folhas de espécies arbóreas nativas da mata atlântica. Dissertação de mestrado. Viçosa, Universidade Federal de Viçosa.

Arato, H.D.; Martins, S.V. \& Ferrari, S.H.S. 2003. Produção e decomposição de serapilheira em um sistema agroflorestal implantado para recuperação de área degradada em Viçosa-MG. Revista Árvore 27: 715-721.

Barlow, J.; Gardner, T.A.; Ferreira, L.V. \& Peres, C.A. 2007. Litter fall and decomposition in primary, secondary and plantation forests in the Brazilian Amazon. Forest Ecology and Management 247: 91-97.

Bataglia, O.C.; Furlani, A.M.C.; Teixeira, J.P.F.; Furlani, P.R. \& Gallo, J.R. 1983. Métodos de análise química de plantas. Campinas, Instituto Agronômico de Campinas. (Boletim Técnico, 78).

Bedford, B.; Walbridge, M. \& Aldous, A. 1999. Patterns in nutrient availability and plant diversity of temperate North American wetlands. Ecology 80(7): 2151-2169.

Berg, B. \& McClaugherty, C. 2007. Plant litter: decomposition, humus formation, carbon sequestration. Berlin Heidelberg, Springer Verlag.

Borém, R.A.T. \& Ramos, D.P. 2002. Variação estacional e topográfica de nutrientes na serapilheira de um fragmento de mata atlântica. Cerne 8(2): 42-59.

Braga, J.M. \& Defelipo, B.V. 1974. Determinação espectrofotométrica de fósforo em extrato de solo e material vegetal. Revista Ceres 21 (1): 73-85.

Chapin, F.; Matson, P. \& Mooney, H. 2002. Principles of terrestrial ecosystem ecology. New York, Springer Verlag.

Cianciaruso, M.V.; Pires, J.S.; Delitti, W.B. \& Silva, E.F. 2006. Produção de serapilheira e decomposição do material foliar em um cerradão na Estação Ecológica de Jataí, município de Luiz Antônio, SP, Brasil. Acta Botanica Brasilica 20(1): 49-59.

Cleveland, C.C.; Reed, S.C. \& Townsend, A.R. 2006. Nutrient regulation of organic matter decomposition in a tropical rain forest. Ecology 87(2): 492-503.

Cuevas, E. \& Lugo, A.E. 1998. Dynamics of organic matter and nutrient return from litterfall in stands of ten tropical tree plantation species. Forest Ecology and Management 112: 263-279.

Davidson, E.A. \& Howarth, R.W. 2007. Nutrients in synergy. Nature 449: 1000-1001.

Dent, D.H.; Bagchi, R.; Robinson, D.; Majalap-Lee, N. \& Burslem, D.F.R.P. 2006. Nutrient fluxes via litterfall and leaf litter decomposition vary across a gradient of soil nutrient supply in a lowland tropical rain forest. Plant Soil 288: 197-215. 
EMBRAPA 1997. Manual de Métodos de Análise de Solo. 2 ed. Rio de Janeiro, Centro Nacional de Pesquisas de Solos.

Fernandes, M.M.; Pereira, M.G.; Magalhães, L.M.; Cruz, A.R. \& Giácomo, R.G. 2006. Aporte e decomposição de serapilheira em áreas de floresta secundária, plantio de Sabiá (Mimosa caesalpiniaefolia Benth.) e Andiroba (Carapa guianensis Aubl.) na FLONA Mário Xavier, RJ. Ciência Florestal 16: 163-175.

Graça, M.A.; Bärlocher, F. \& Gessner, M. 2005. Methods to study litter decomposition. Dordrecht, Springer Verlag.

Greenland, D.J. \& Kowal, J,M.L. 1960. Nutrient content of the moist tropical forest of Ghana. Plant and Soil 12: 154-173.

Güsewell, S. 2004. N: P ratios in terrestrial plants: variation and functional significance. New Phytologist 164: 243-266.

Haase, R. 1999. Litterfall and nutrient return in seasonally flooded and non flooded forest of the Pantanal, Mato Grosso, Brazil. Forest Ecology and Management 117: 129-147.

Harms, W.; H. Schreuder; D. Hook \& Brown, C. 1980. The effects of flooding on the Swamp Forest in Lake Ocklawaha, Florida. Ecology 61: 1412-21.

Hobbie, S.E. \& Vitousek, P.M. 2000. Nutrient Limitation of Decomposition in Hawaiian Forests. Ecology 81(7): 1867-1877.

Hora, R.C.; Primavesi, O. \& Soares, J.J. 2008. Contribuição das folhas de lianas na produção de serapilheira em um fragmento de floresta estacional semidecidual em São Carlos, SP. Revista Brasileira de Botânica 31(2): 277-285.

Junior, L.R.; Fisch, S.T. \& Ballestero, S.D. 2003. Influência da umidade do solo no desenvolvimento inicial de plantas do palmiteiro Euterpe edulis Mart. em floresta nativa. Revista Biociências 9(1): 7-13.

Killingbeck, K.T. 1996. Nutrients in senesced leaves: keys to the search for potential resorption and resorption proficiency. Ecology 77: 1716-1727.

König, F.G.; Brun, E.J.; Schumacher, M.V. \& Jonas, L. 2002. Devolução de Nutrientes via serapilheira em um fragmento de floresta estacional decidual no município de Santa Maria, RS. Brasil Florestal 74: 45-52.

Kozovits, A.R.; Bustamante, M.M.; Garofalo, C.R.; Bucci, S.; Franco, A.C. \& Goldstein, G. 2007. Nutrient resorption and patterns of litter production and decomposition in a Neotropical Savanna. Functional Ecology 21: 1034-1043.

Larcher, W. 2000. Ecofisiologia vegetal. São Carlos, Rima.

Luizão, R.C.C.; Luizão, F.J.; Paiva, R.Q.; Monteiro, T.F.; Sousa, L.S. \& Kit\$, B. 2004. Variation of carbon and nitrogen cycling processes along a topographic gradient in a central amazonian forest. Global Change Biology 10: 592-600.

Martins, S.V. \& Rodrigues, R.R. 1999. Produção de serapilheira em clareiras de uma floresta estacional semidecidual no município de Campinas, SP. Revista Brasileira de Botânica 22(3): 405-412.

Montagnini, F. \& Jordan, C.F. 2005. Tropical forest ecology: the basis for conservation and management. Berlin Heidelberg, Springer-Verlag.

Moraes, R.M.; Delitti, W.B.C. \& Struffaldi-De Vuono, Y. 1999 Litterfall and litter nutrient content in two Brazilian Tropical Forests. Revista Brasileira de Botânica 22(1): 9 -16.

Pagano, S.N. \& Durigan, G. 2000. Aspectos da ciclagem de nutrientes em Matas Ciliares do oeste do Estado de São Paulo, Brasil. Pp. 109-123.
In: Matas ciliares: conservação e recuperação. São Paulo, Editora USP/FAPESP.

Pedreira, G.L. 2008. Avaliação do Regime hídrico, geológico e geomorfológico das florestas paludosas do Parque Estadual do Itacolomi: influencia dos fatores abióticos sobre a composição floristica e fitossociológica. Dissertação de mestrado. Departamento de Geologia, Universidade Federal de Ouro Preto. Ouro Preto, MG.

Pinto, S.I.C.; Martins, S. V.; Barros, N.F. \& Dias, H.C.T. 2009. Ciclagem de nutrientes em dois trechos de floresta estacional semidecidual na reserva florestal Mata do Paraíso em Viçosa, MG, Brasil. Revista Árvore 33(4): 653-663.

Pinto, S.I.; Martins, S.V. \& Dias, H.C. 2008. Produção de serapilheira em dois estádios sucessionais de floresta estacional semidecidual na Reserva Mata do Paraíso, em Viçosa, MG. Revista Árvore 32(3): 545-556.

Ramos, M.V.V.; Curi, N.; Motta, P.E.F.; Vitorino, A.C.T.; Ferreira, M.M. \& Silva, M.L.N. 2006. Veredas do Triângulo Mineiro: solos, água e uso. Ciência e Agrotecnologia 30(2): 283-293.

Richardson, J. \& Vepraskas, M.J. 2001. Wetland Soils: Genesis Hydrology Landscapes and Classification. New York, Lewis Publishers.

Scheer, B. 2008. Decomposição e liberação de nutrientes da serapilheira foliar em um trecho de floresta ombrófila densa aluvial em regeneração, Guaraqueçaba (PR). Revista Floresta 38(2): 253-266.

SEMAD/ IEF/ PROMATA. 2007. Plano de Manejo do Parque Estadual do Itacolomi. Encarte 1.

Taiz, L. \& Zeiger, E. 2004. Fisiologia Vegetal. Porto Alegre, Artmed.

Teixeira, A.P. \& Assis, M.A. 2005. Caracterização florística e fitossociológica do componente arbustivo-arbóreo de uma floresta paludosa no Município de Rio Claro (SP), Brasil. Revista Brasileira de Botânica 28(3): 467-476.

Toniato, M.T.Z.; Leitão Filho, H.F. \& Rodrigues, R.R. 1998. Fitossociologia de um remanescente de floresta higrófila (mata de brejo) em Campinas, SP. Revista Brasileira de Botânica 21: 197-210.

Torres, R.B; Matthes, L.A.F. \& Rodrigues, R.R. 1994. Florística e estrutura do componente arbóreo de mata de brejo em Campinas, SP. Revista Brasileira de Botânica 17: 189-194.

Vasconcelos, H.L. \& Luizão, F.J. 2004. Litter production and litter nutrient concentrations in a fragmented amazonian landscape. Ecological Applications 14(3): 884-892.

Villela, D.M. \& Proctor, J. 1999. Litterfall mass, chemistry and nutrient retranslocation in a monodominant forest on Maracá Island, Roraima, Brazil. Biotropica 31(2): 198-211.

Villela, D.M. \& Proctor, J. 2002. Leaf litter decomposition and monodominance in the Peltogyne forest of Maracá Island, Brazil. Biotropica 34(3): 334-347.

Vital, A.R.T; Guerrini, I.A.; Franken, W.K. \& Fonseca, R.C.B. 2004. Produção de serapilheira e ciclagem de nutrientes de uma floresta estacional semidecidual em zona ripária. Revista Árvore 28(6): 793-800.

Vitousek, P.M. 1982. Nutrient cycling and nutrient use efficiency. The American Naturalist 119: 553-572.

Vitousek, P.M. 1984. Litterfall, nutrient cycling and nutrient limitation in tropical forests. Ecology 65(1): 285-298.

Vitousek, P.M. \& Sanford Jr, R.L. 1986. Nutrient cycling in moist tropical forest. Annual Review of Ecology and Systematics 17: 137-167. 